\title{
Down regulation of lincRNA-p21 contributes to gastric cancer development through Hippo-independent activation of YAP
}

\author{
Ying Chen ${ }^{1, *}$, Guoqing Wei ${ }^{2, *}$, Hongwei Xia ${ }^{2}$, Huangfei $\mathbf{Y u}^{1}$, Qiuling Tang ${ }^{2}$ and \\ Feng $\mathrm{Bi}^{1,2}$ \\ ${ }^{1}$ Department of Medical Oncology, West China Hospital, Sichuan University, Chengdu, P.R. China \\ ${ }^{2}$ State Key Laboratory of Biotherapy and Cancer Center, West China Hospital, Sichuan University, and Collaborative Innovation \\ Center for Biotherapy, Chengdu, P.R. China \\ *These authors have contributed equally to this work \\ Correspondence to: Feng Bi, email: bifeng@medmail.com.cn \\ Keywords: long non-coding RNA, lincRNA-p21, gastric cancer, YAP, hippo \\ Received: August 23, $2016 \quad$ Accepted: June 13,2017 Published: July 10, 2017 \\ Copyright: Chen et al. This is an open-access article distributed under the terms of the Creative Commons Attribution License 3.0 \\ (CC BY 3.0), which permits unrestricted use, distribution, and reproduction in any medium, provided the original author and source \\ are credited.
}

\section{ABSTRACT}

Long intergenic non-coding RNA p21 (lincRNA-p21), known as the direct transcriptional target of p53, was found down-regulated in several human solid tumors. However, little is known about the role of lincRNA-p21 in gastric cancer. The expression levels of lincRNA-p21 in tissue samples and cell lines were detected by qRTPCR. MGC-803 and MKN-45 cells were transfected with siRNAs targeting lincRNA-p21 or control siRNAs to determine the effect of reduced lincRNA-p21 expression on tumorigenesis. We also overexpressed lincRNA-p21 in MGC-803 cells. Cell proliferation was measured by CCK-8 and Ethynyl-2-deoxyuridine (EdU) incorporation assays. Migration and invasion abilities of cells were measured by wound healing and transwell assay. We demonstrated that lincRNA-p21 was significantly reduced in gastric cancer tissues $(p<0.001)$ compared with that in normal tissues and this lower level of lincRNA-p21 was significantly correlated with higher invasion depth grade $(p=0.024)$, more distant metastasis $(p=0.009)$ and advanced TNM stage $(p=0.011)$. Further study revealed that knock down of lincRNA-p21 could promote malignant behavior of gastric cancer cells and induce epithelial to mesenchymal transition (EMT). Overexpressing lincRNA-p21 showed opposite effects. Moreover, knocking down lincRNA-p21 could elevate the expression of Yes associated protein (YAP), the core effector of Hippo signaling, by elevating mRNA levels and increasing its nucleus translocation instead of the canonical Hippo pathway. Overexpression experiments verified the regulation role of lincRNA-p21 in YAP expression. Collectively, these data suggest that lincRNA-p21 could serve as a potential biomarker and a vital therapeutic target in gastric cancer.

\section{INTRODUCTION}

Gastric cancer (GC) is the most common gastrointestinal cancer in East Asia and ranks as the third leading type of cancer-related death worldwide [1, 2]. The mortality rate has remained at a rather high level during the latest 10 years in China [3, 4]. Therefore it is urgent that we identify novel biomarkers and their underlying molecular mechanisms in gastric carcinogenesis. These biomarkers may not only improve the early diagnosis and prognosis, but also may serve as molecular targets for gastric cancer therapy.

Long noncoding RNA (lncRNA), which is defined as transcripts composed of more than 200 nucleotides in length, have attracted broad attention recently [5-7]. Long intergenic non-coding RNA p21 (lincRNA-p21) is known 
as a direct transcriptional target of p53 and is $\sim 3 \mathrm{~kb}$ in length. It mainly regulates the cell cycle and apoptosis [8]. Several studies have shown that lower expression levels of lincRNA-p21 are correlated with the poor prognosis of colorectal cancer and hepatocellular carcinoma [9, 10]. However, the role of lincRNA-p21 in gastric cancer remains largely unknown.

The Hippo pathway governs the organ size and cell growth rate [11]. Yes-associated protein (YAP), the core effector of Hippo pathway, has long been considered an important proto-oncogene. The overexpression of YAP was found in multiple types of malignancies, including liver cancer, esophageal squamous cell carcinoma, non-small lung cancer and ovarian cancer $[12-15,18]$. Although the regulation of YAP by the Hippo pathway has been heavily researched, how YAP is regulated by Hippo-independent pathways remains elusive.

Our study first confirmed the expression pattern of the lincRNA-p21 expression in GC tissues and cell lines. We then investigated the relationship between lincRNA-p21 levels and the malignant behavior of gastric cancer in two GC cell lines. The potential lincRNA-p21-dependent mechanisms correlated with EMT and YAP expression were further explored using in vitro experiments.

\section{RESULTS}

\section{LincRNA-p21 is significantly down-regulated in gastric cancer}

We first measured the expression level of lincRNA-p21 by qRT-PCR in 40 pairs of gastric cancer tissues and adjacent non-cancerous tissues. We first expressed this paired group data as fold changes in the GC tissues relative to the paired normal tissues and then separated the paired tissues into low $(n=21$, ratio $\leq 0.11)$ and high $(\mathrm{n}=19$, ratio $>0.11)$ expression groups according to the median ratio (Figure 1A). Our statistical analysis indicated that lincRNA-p21 was significantly downregulated in tumor tissue samples compared to paired normal tissue $(p<0.05)$ (Figure $1 \mathrm{C})$. Further analysis indicated a significantly lower level of lincRNA-p21 in GC tissues with distant metastasis $(p<0.001)$ (Figure 1D). However, the reduced lincRNA-p21 expression was not significantly correlated with lymph node metastasis $(p>0.05)$ (Figure 1E). The comparison of the clinicopathological characteristics between relative low expression group and high expression group demonstrated that the lower expression level of lincRNA-p21 was correlated with higher invasion depth grade $(p=0.024)$,
A

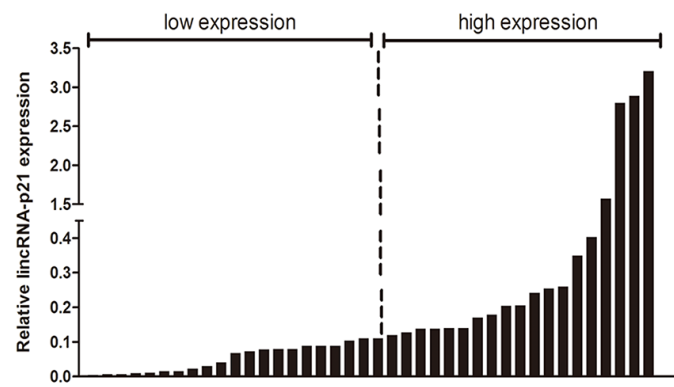

B

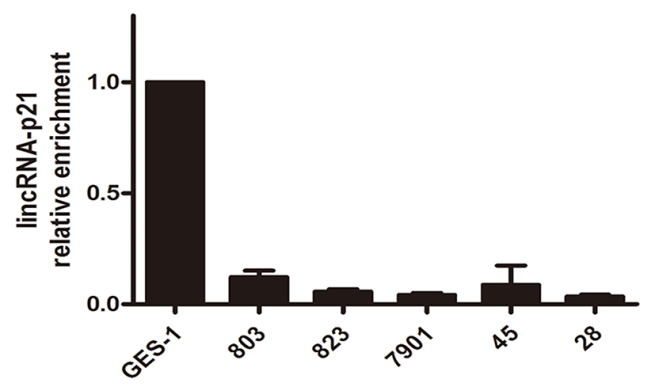

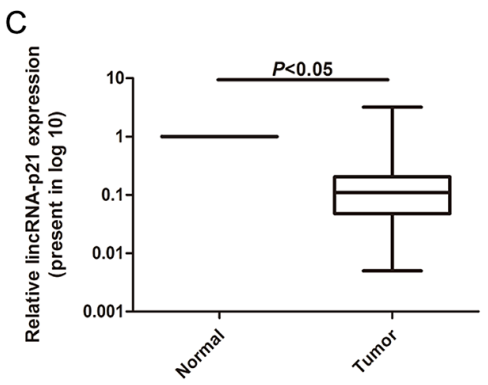
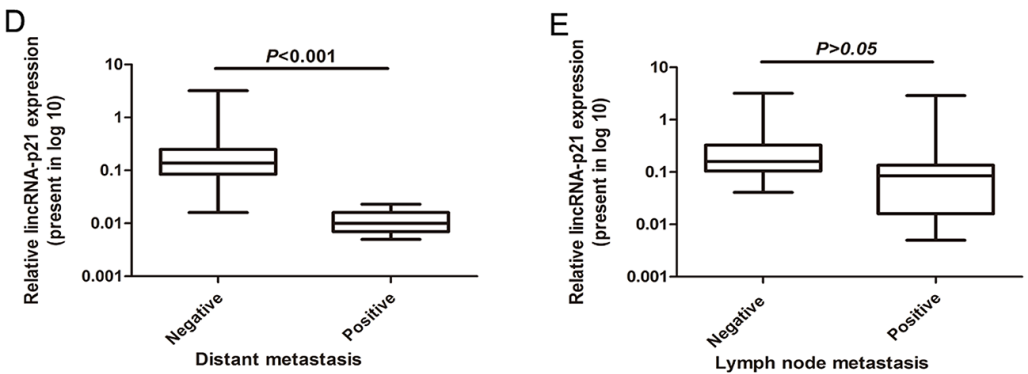

Figure 1: The expression level of lincRNA-p21 in GC tissues and GC cell lines. The relative lincRNA-p21 expression level was measured by qRT-PCR (A) The data was shown as the fold change in the GC tissues compared with the paired normal tissues. Two groups were set according to the median ratio. (B) The lincRNA-p21 expression in GC cell and GES-1. (C) The statistical analysis of the lincRNA-p21 expression in GC tissues compared to the paired normal tissues. (D) The relationship betweenlincRNA-p21 expression and distant metastasis. (E) The relationship between lincRNA-p21 expression andlymph node metastasis. Bars represents for Standard Deviation (SD). All data showed a representative of three independent experiments. 
Table 1: Correlation between lincRNA-p21 expression and clinicopathological features in gastric cancer patients

\begin{tabular}{|c|c|c|c|c|}
\hline \multirow[t]{2}{*}{ Clinical characteristics } & \multirow[t]{2}{*}{ Total number } & \multicolumn{2}{|c|}{ LincRNA-p21 expression } & \multirow[t]{2}{*}{$P$ value } \\
\hline & & low & high & \\
\hline \multicolumn{5}{|l|}{ Age } \\
\hline$>60$ & 18 & 7 & 11 & 0.107 \\
\hline$<60$ & 22 & 14 & 8 & \\
\hline \multicolumn{5}{|l|}{ Gender } \\
\hline Male & 25 & 13 & 12 & 0.597 \\
\hline Female & 15 & 8 & 7 & \\
\hline \multicolumn{5}{|l|}{ Invasion depth } \\
\hline $\mathrm{T} 1$ & 4 & 1 & 3 & $0.024 *$ \\
\hline $\mathrm{T} 2$ & 11 & 3 & 8 & \\
\hline $\mathrm{T} 3$ & 12 & 6 & 6 & \\
\hline $\mathrm{T} 4$ & 13 & 11 & 2 & \\
\hline \multicolumn{5}{|l|}{ Lymphnode metastasis } \\
\hline 0 & 16 & 5 & 11 & 0.057 \\
\hline 1 & 13 & 7 & 6 & \\
\hline 2 & 7 & 5 & 2 & \\
\hline 3 & 4 & 4 & 0 & \\
\hline \multicolumn{5}{|l|}{ Metastasis } \\
\hline Yes & 7 & 7 & 0 & $0.009^{*}$ \\
\hline No & 33 & 14 & 19 & \\
\hline \multicolumn{5}{|l|}{ TNM stage } \\
\hline I & 13 & 3 & 10 & $0.011^{*}$ \\
\hline II & 12 & 6 & 6 & \\
\hline III & 8 & 5 & 3 & \\
\hline IV & 7 & 7 & 0 & \\
\hline
\end{tabular}

*represents for $\mathrm{p}<0.05$

distant metastasis $(p=0.009)$ and more advanced TNM stage $(p=0.011)$ (Table 1$)$. Next, we performed qRT-PCR to examine the expression of lincRNA-p21 in gastric cancer cell lines. The results showed that lincRNA-p21 was significantly decreased in multiple gastric cancer cell lines compared with the normal gastric mucosal cell line GES-1 (Figure 1B).

\section{The effect of lincRNA-p21 on GC cell proliferation}

In order to study the biological function of lincRNA-p21 in gastric cancers, we performed knockdown and overexpression experiments. The expression level of lincRNA-p21 was significantly down-regulated in the
si-lincRNA-p21-transfected cells ( $p<0.05$, Figure 2A). Meanwhile, the expression level of lincRNA-p21 was significantly up-regulated in the cells transfected with a pcDNA 3.1-lincRNA-p21 overexpresssion vector $(p<0.01$, Figure 2B). CCK-8 assays demonstrated that the knockdown of lincRNA-p21 promoted cell growth in both MGC-803 and MKN-45 cells (Figure 2C) while lincRNA-p21 overexpression showed the opposite result (Figure 2D). Additionally, the Edu assay also showed enhanced cell proliferation ability in the MGC-803 (Figure 2E and 2F) and MKN-45 cells (Figure 2G and 2H) transfected with si-lincRNA-p21. In contrast, the cell proliferation ability of MGC-803 was impaired during lincRNA-p21 overexpression (Figure 2I and 2J). 


\section{The relationship between lincRNA-p21 and}

\section{EMT process}

We next evaluated the effect of down-regulated lincRNA-p21 on the EMT process. We found that the morphology of both MGC-803 and MKN-45 dramatically changed at $48 \mathrm{~h}$ post-transfection of silincRNA-p21. They lost their cell to cell contacts and acquired spindle-like appearance (Figure 3A). Our western blots showed that vimentin and $\mathrm{N}$-cadherin were significantly elevated in si-lincRNA-p21 group
(Figure 3B) while lincRNA-p21 overexpression showed an opposite result (Figure 3C). In wound healing assays, we showed that the wound area became narrower in silincRNA-p21 groups compared with control groups in both MGC-803 (Figure 3D) and MKN-45 (Figure 3E) cells. Quantitative analysis revealed that the decrease of the wound area was statistically significant at both $24 \mathrm{~h}$ and $48 \mathrm{~h}$ post-transfection time point in MGC-803 (Figure 3F) and MKN-45 (Figure 3G) cells. Transwell assays were also performed to test the cell migration and invasion ability. We observed that the knockdown
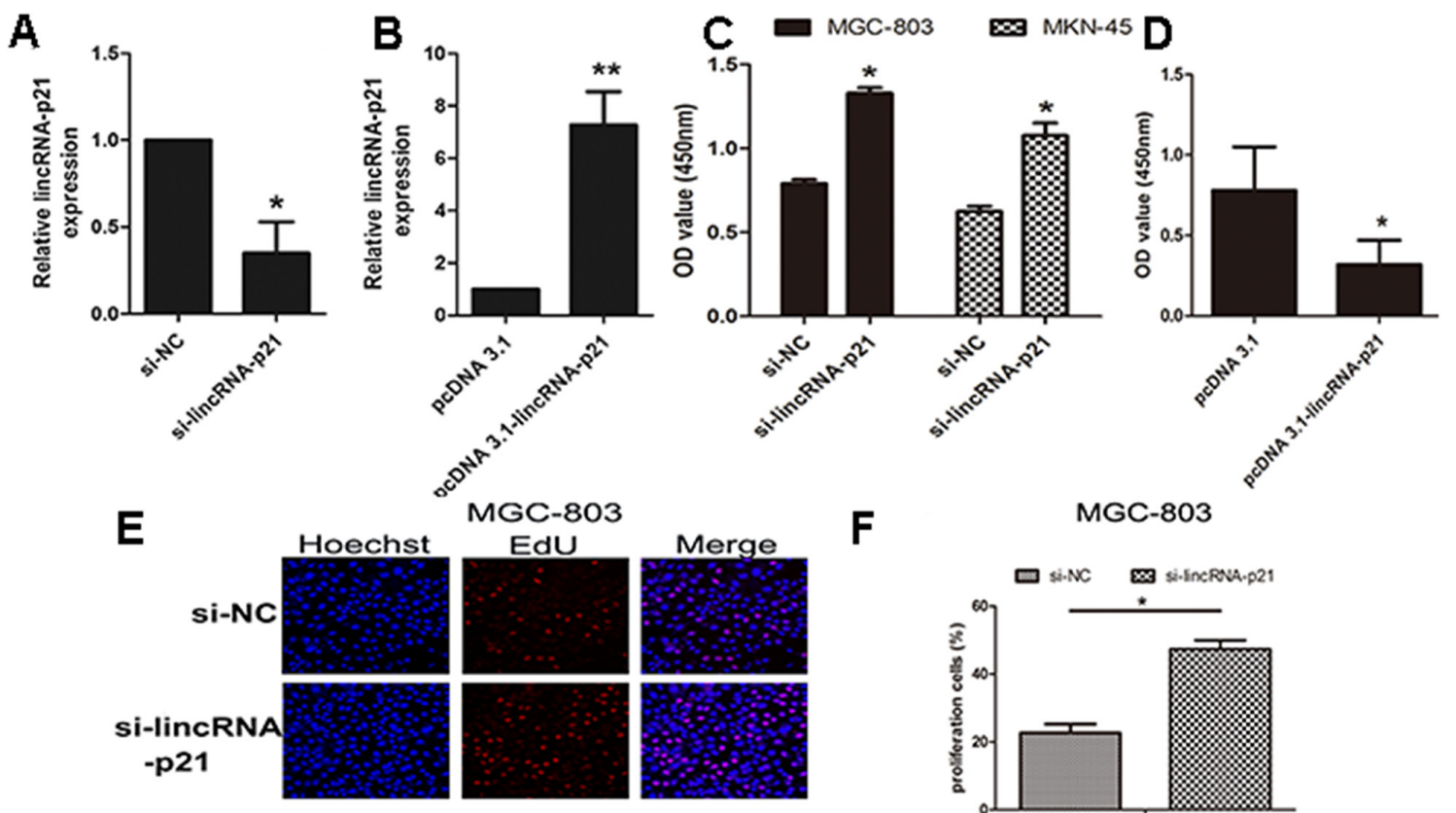

$\mathbf{F}$

MGC-803
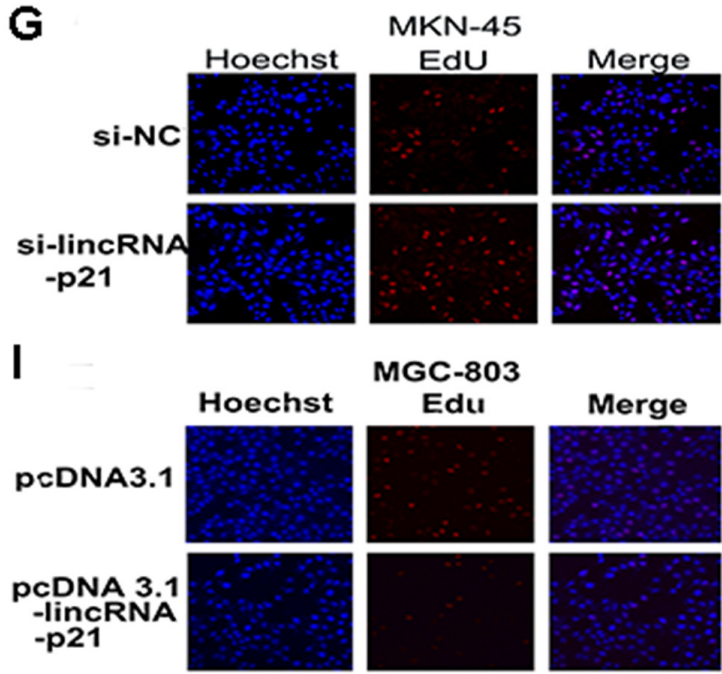

H
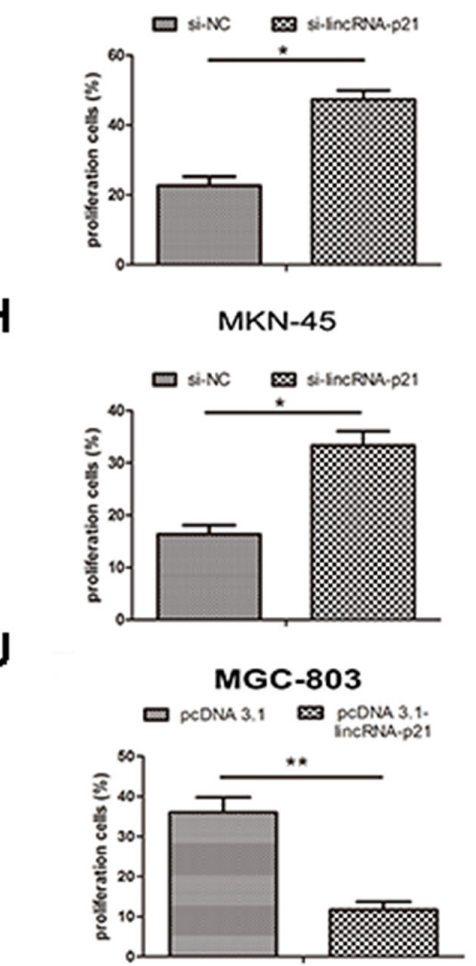

Figure 2: The effect of lincRNA-p21 on GC cell proliferation. The knockdown efficiency (A) and overexpression (B) efficiency of lincRNA-p21 tested in MGC-803. CCK-8 kit was used to test the cell viability at 48h post-transfection of siRNA in both MGC-803 and MKN-45 (C). CCK-8 data of pcDNA 3.1-lincRNA-p21 transfection in MGC-803 cell line (D). Edu incorporation assay was performed to further test the cell proliferation at 48h post-transfection. Pictures of knockdown experiments in MGC-803 (E) and MKN-45 (G). Pictures of overexpression experiments in MGC-803 cells (I). Red labeled cells indicated as the proliferated cells. The percentage of the proliferated cells was also counted in both knockdown experiments $\mathbf{( F )}$ and $\mathbf{( H )}$ and overexpression experiments $(\mathbf{J})$. Bars represents for SD. All data shows a representative of three independent experiments. * represents for $p<0.05, * *$ represents for $p<0.01$. 
of lincRNA-p21 lead to more cells passing through the chambers with or without the coated matrigel in MGC803 and MKN-45 cells (Figure 4A and 4C). Further quantitative analysis showed a significantly increased number of cells passing through the chamber in MGC803 and MKN-45 experiments during knockdown of lincRNA-p21 (Figure 4B and 4D). In lincRNA-p21 overexpression experiments, fewer cells passed through the chambers compare to control groups (Figure 4E) and quantitative analysis supported this finding (Figure 4F).

\section{The role of lincRNA-p21 in the regulation of} YAP expression

We tested the expression level of several vital proteins in the GC cells transfected with si-lincRNA-p21. Our result showed up-regulated YAP, $\beta$-catenin and NF$\kappa \mathrm{B}$ in the si-lincRNA-p21 group, which is consistent with several recent reports $[22,23]$, while the expression of P-ERK was somehow decreased (Figure 5A). We found up-regulated YAP and P-YAP level in the lincRNA-p21
A
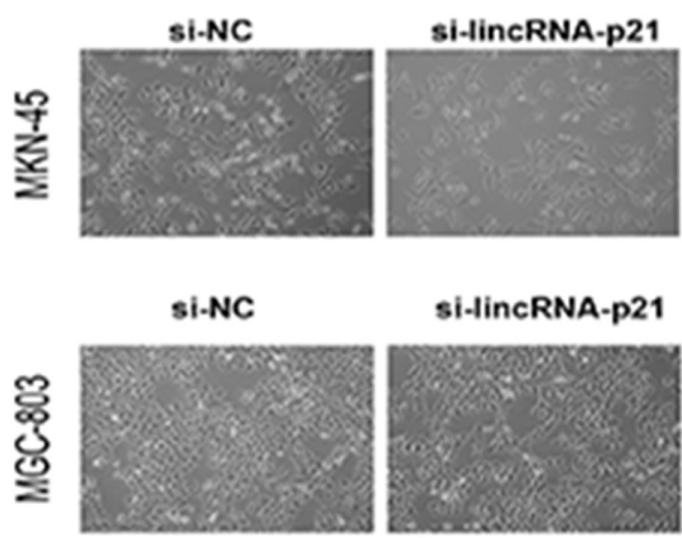

D
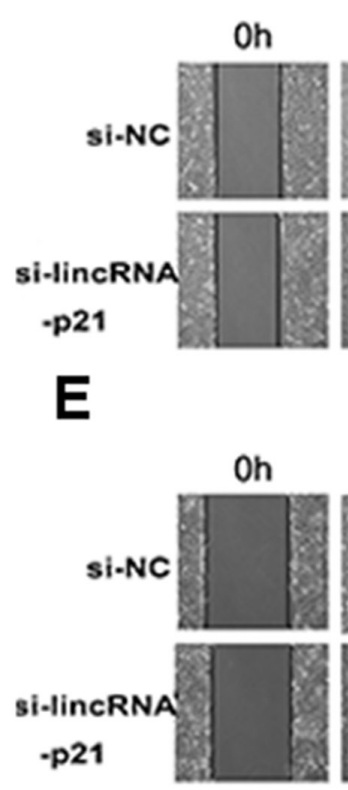

si-lincRNA-p21

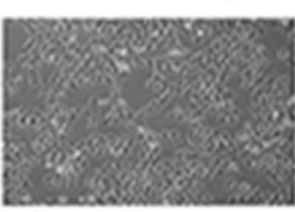

MGC-803 $24 \mathrm{~h}$
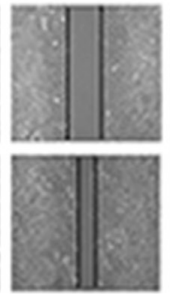

MKN-45 $24 \mathrm{~h}$

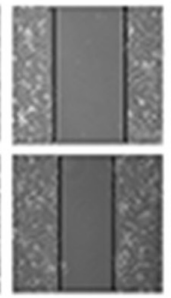

$48 \mathrm{~h}$
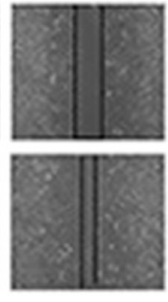

$48 \mathrm{~h}$

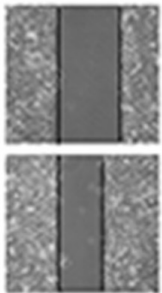

B

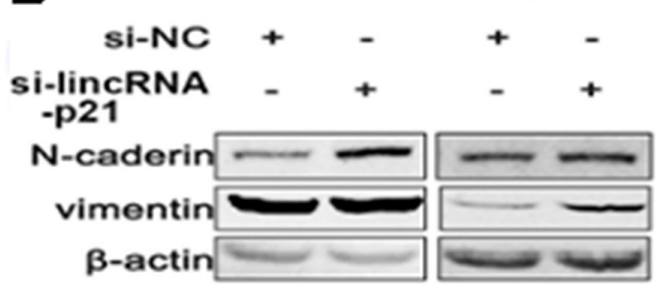

Q

MGC-803

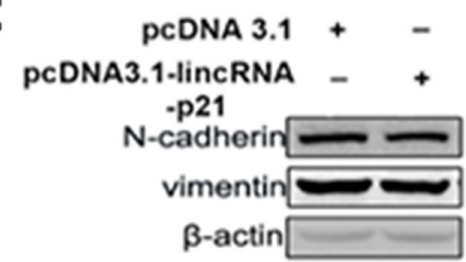

F MGC-803

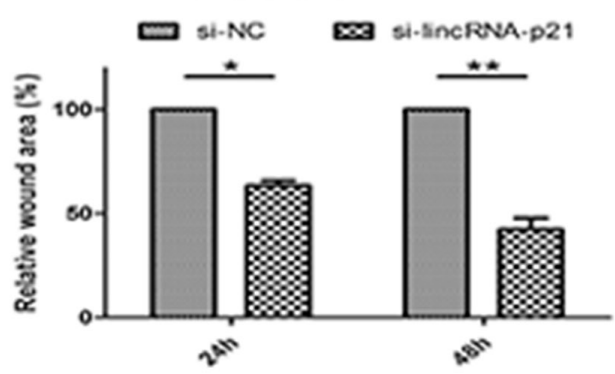

G

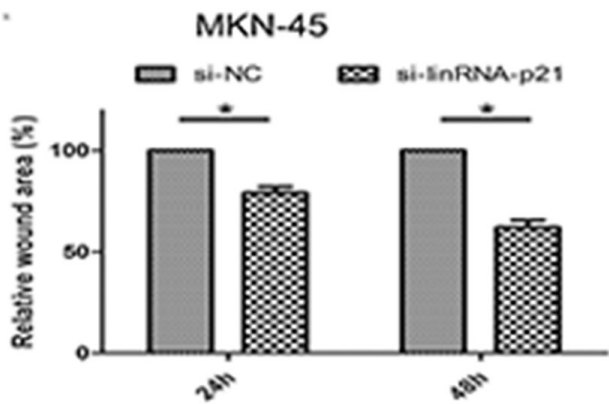

Figure 3: The effect of lincRNA-p21 on cell migration ability and EMT process. The morphology change was observed at 48h post-transfection of siRNA in MGC-803 and MKN-45 cell lines by bright field microscopy (20×) (A). The western blot result in knockdown experiment (B) and overexpression experiment (C). Pictures of wound healing assay in knockdown experiments (D) and (E). Relative wound area was calculated as (wound area at 24 or $48 \mathrm{~h} /$ wound area at $0 \mathrm{~h}$ ), which was then normalized to si-NC group in MGC-803 (F) and MKN-45 (G). Bars represents for SD. All data shows a representative of three independent experiments. * represents for $\mathrm{p}<0.05, * *$ represents for $\mathrm{p}<0.01$. 
knockdown cells (Figure 5B). However, the expression of LATS1, the main upstream activator of YAP cytoplasmic accumulation in Hippo pathway, remained almost unchanged (Figure 5B). Next qRT-PCR assays showed that the down-regulated lincRNA-p21 expression correlated with elevated levels of YAP mRNA, connective tissue growth factor (CTGF) and cysteine-rich angiogenic inducer 61 (CYR-61), in MGC-803 cells (Figure 5C) and MKN-45 cells (Figure 5D). CTGF and CYR-61 are two targets of YAP activation [24]. Moreover, we also found lower levels of YAP and P-YAP expression in the MGC803 cells transfected with pcDNA 3.1-lincRNA-p21 (Figure 5E). Furthermore, qRT-PCR assays also showed decreased mRNA levels of YAP, CTGF and CYR-61 in MGC-803 cells transfected with pcDNA 3.1-lincRNA-p21 (Figure 5F).

\section{The regulation of lincRNA-p21 on YAP nuclear translocation}

Besides the elevated mRNA of YAP, we wondered whether the elevated YAP protein level was correlated with increased YAP nuclear translocation. We first extracted nuclear and cytoplasmic proteins separately and analyzed them by western blot. We found increased YAP protein levels in both the nucleus and cytoplasm in two GC cell lines transfected with si-lincRNA-p21 (Figure 6A and 6B). Overexpression of linRNA-p21 showed an opposite result (Figure 6C). We also performed immunofluorescence staining experiments to visualize YAP intracellular localization. Staining revealed an obvious accumulation of YAP in the nucleus in si-lincRNA-p21 treated-cells compared to control siRNA-treated cells, in which YAP
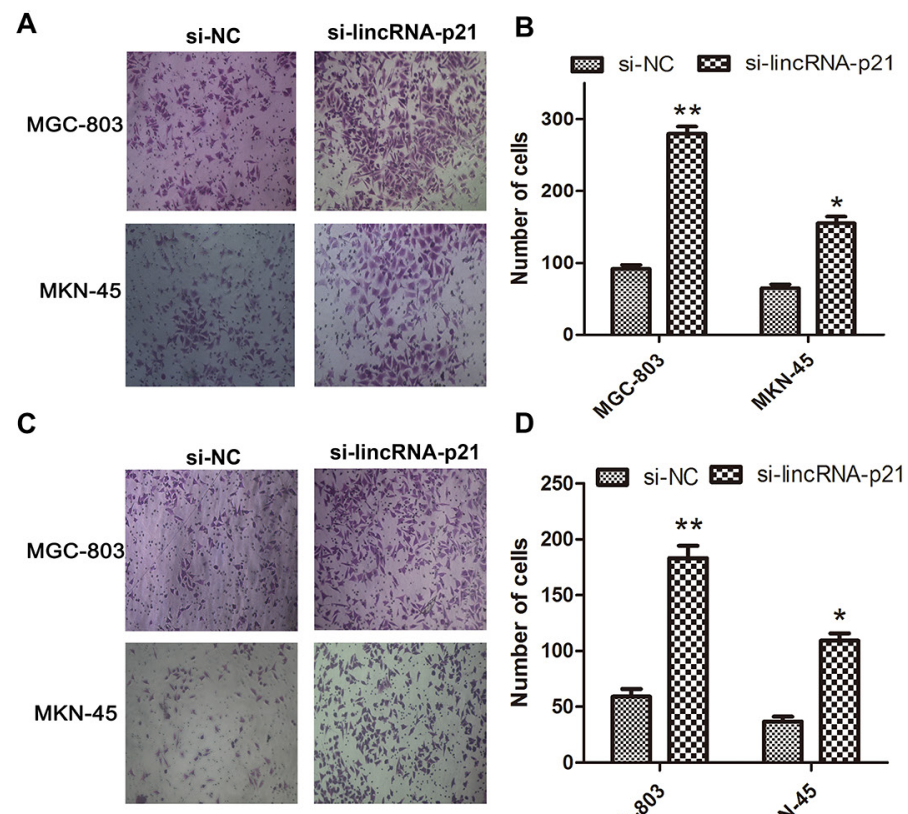

D
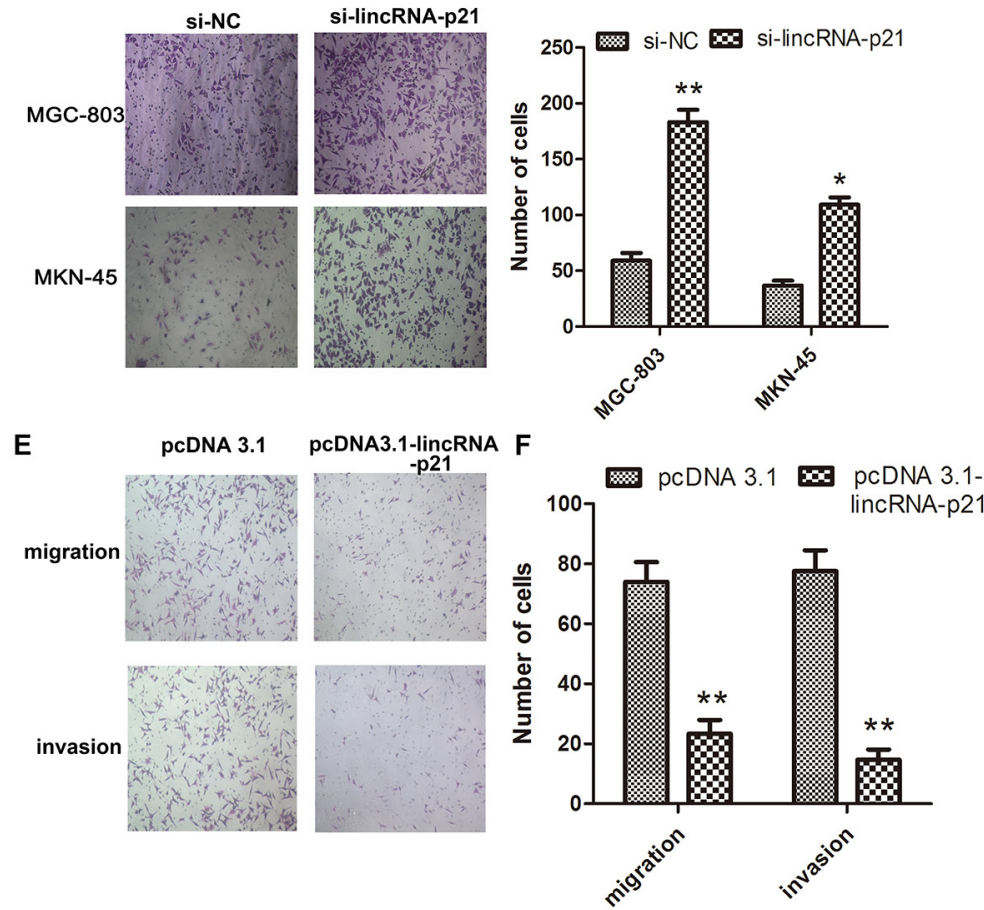

Figure 4: The effect of lincRNA-p21 on cell migration and invasion ability. Representative pictures of transwell assays showed the change of thecell migration (A) and invasion (C) ability in knockdown experiments and in overexpression experiments (E). Quantification data in knockdown experiments (B) and (D) as well as overexpression experiments were showed (F). Bars represents for SD. All data shows a representative of three independent experiments. * represents for $\mathrm{p}<0.05$, ** represents for $\mathrm{p}<0.01$. 
was mainly cytoplasmic (Figure 6D and 6E). We conclude that lincRNA-p21 may regulate YAP nuclear translocation.

\section{The involvement of lincRNA-p21 in the GC development may relate to the YAP expression}

Since YAP plays an important role in tumorigenesis and triggering the EMT process [16, 17], we wondered whether the involvement of lincRNA-p21 in the GC development was related to the YAP expression. The knockdown efficiency of si-YAP was tested by both qRTPCR (Figure 7A) and western blot (Figure 7B). Western blots indicated that cells transfected with siRNAs targeting both lincRNA-p21 and YAP counteracted the induction of YAP, N-cadherin, Vimentin and C-myc observed in single si-lincRNA-p21 transfection group in both
MGC-803 (Figure 7C) and MKN-45 (Figure 7D) cells. Overexpression experiments validated these observations (Figure 7E). In this case, we came to the conclusion that the down-regulation of lincRNA-p21 contributes to GC development, possibly through the upregulation of YAP.

\section{DISCUSSION}

The high incidence rate and relative low survival rate makes gastric cancer a significant threat to human health. However, GC is a multistep carcinogenesis process which involves genetic alterations, epigenetic changes and multiple oncogenic pathways. Thus understanding the mechanism of $\mathrm{GC}$ development is urgent for us to discover new potential targets for effective therapy.

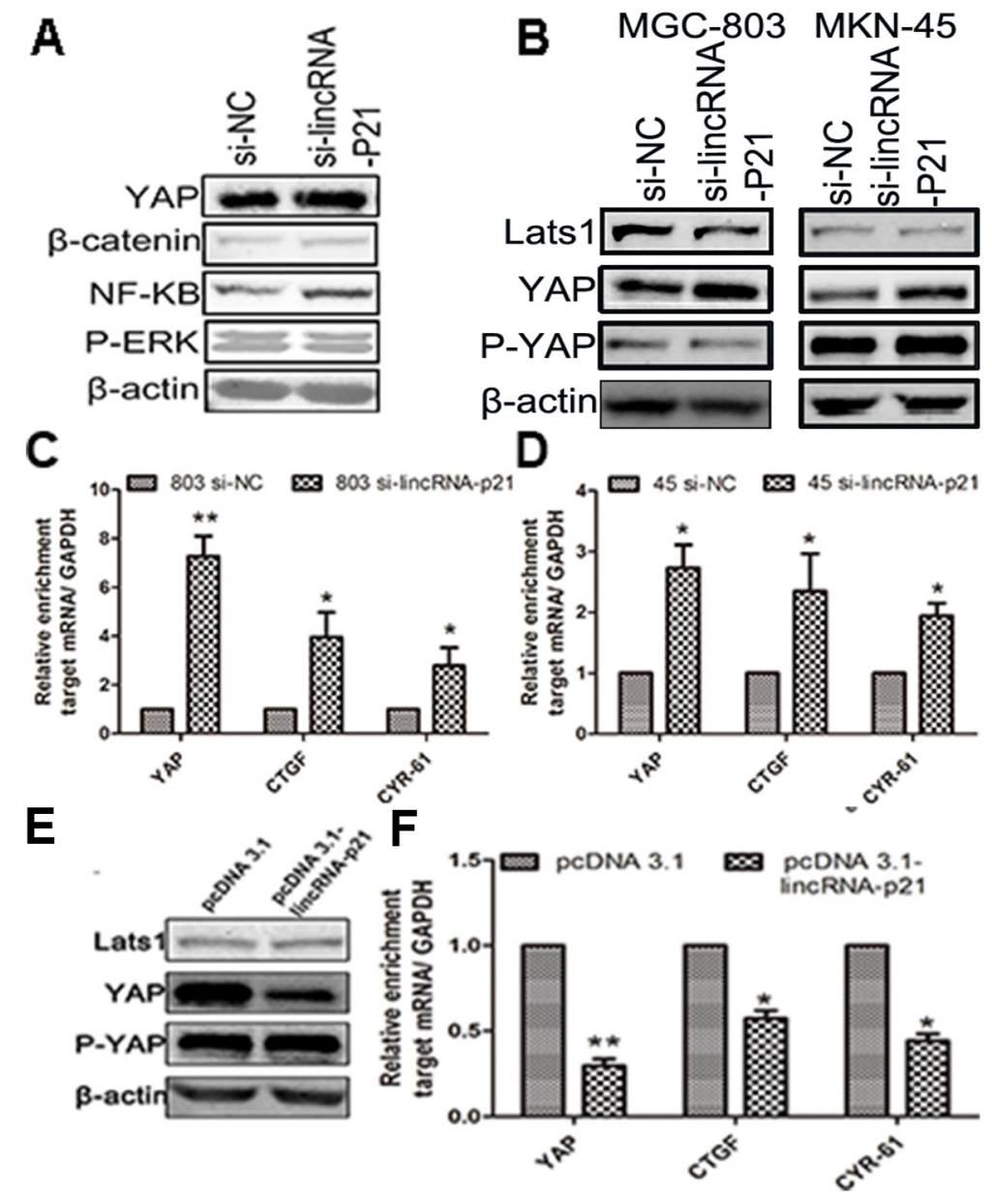

Figure 5: The regulation of lincRNA-p21 on YAP expression was performed in a Hippo independent manner. (A) The western blot showed elevated expression of $\beta$-catenin, YAP and NF- $\kappa B$ in si-lincRNA-p21 group. (B) The western blot result indicated that the YAP and P-YAP were up-regulated in si-lincRNA-p21 group while the Lats1 remained almost unchangeable. Knocking down of lincRNA-p21 resulted in the elevation of mRNA levels of YAP, CTGF and CYR-61 in (C) and MKN-45 (D) cells. (E) The western blot result showed that the YAP and P-YAP were down-regulated in MGC-803 cells transfected with pcDNA 3.1-lincRNA-p21 while the Lats 1 still remains unchangeable. (F) Decreased mRNA level of YAP, CTGF and CRY-61 were observed in overexpression experiment. Bars represents for SD. All data shows a representative of three independent experiments. * represents for $\mathrm{p}<0.05$, ** represents for $\mathrm{p}<0.01$. 
LncRNAs have become focal points in studying cancer development and progression due to their extensive regulation in cells and roles as signals, decoys, guides and scaffolds [25, 26]. LincRNA-p21 was first described by the Huarte lab as a tumor suppressive noncoding RNA with a length of $\sim 3 \mathrm{~kb}$. It could regulate the cell cycle and apoptosis as a direct transcriptional target of p53 [8]. Since abnormal p53 gene status contributes to the progression of various tumors, the involvement of lincRNA-p21 in carcinogenesis has attracted much attention. During the analysis of the expression pattern of lincRNA-p21 in GC tissues, we found a significantly decreased lincRNA-p21 level in the gastric tissues compared with normal tissues. What's more, the clinicopathological feature analysis revealed that the lower level of

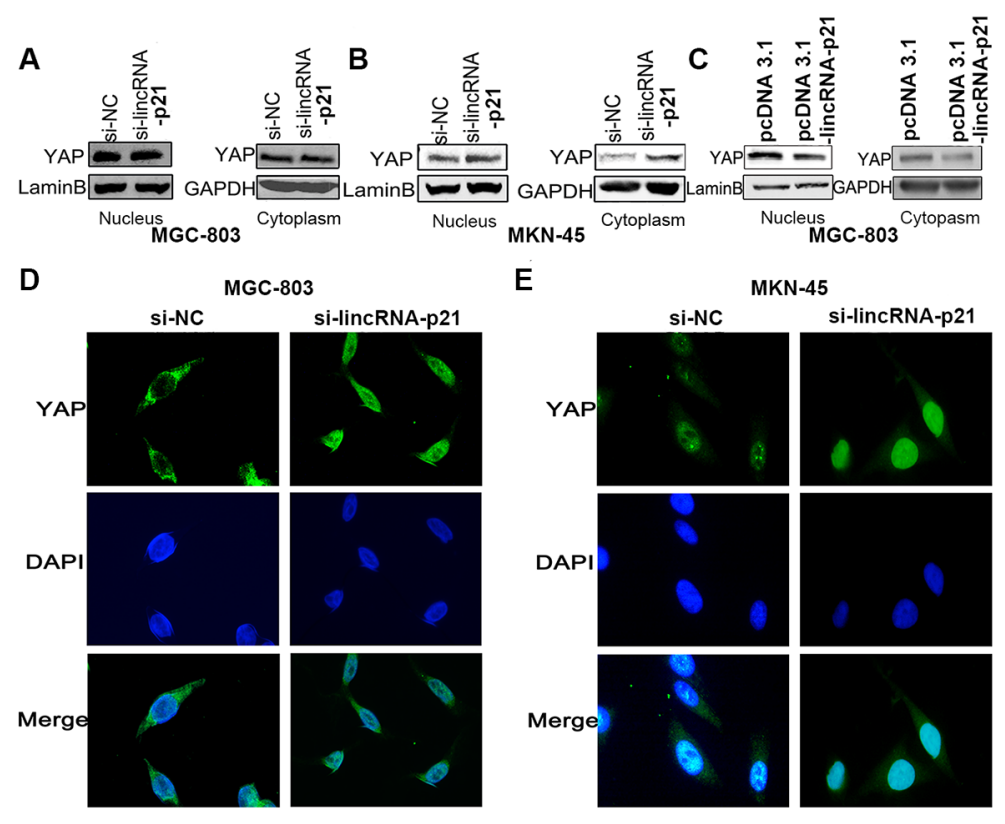

Figure 6: The regulation of lincRNA-p21 on YAP expression might exert through nucleus translocation. The cell lysates from nucleus and cytoplasm were subjected to western-blot. In knockdown experiments, overexpression of YAP in both nucleus and cytoplasm were found in MGC-803 (A) and MKN-45 cells (B). In overexpression experiment, down-regulated YAP expression was observed in nucleus and cytoplasm in MGC-803 cells (C). IF staining demonstrated an obvious nucleus accumulation of YAP in cells transfected with si-lincRNA-p21 in MGC-803 (D) and MKN-45 (E) cells. Pictures were captured under the 40 magnification.

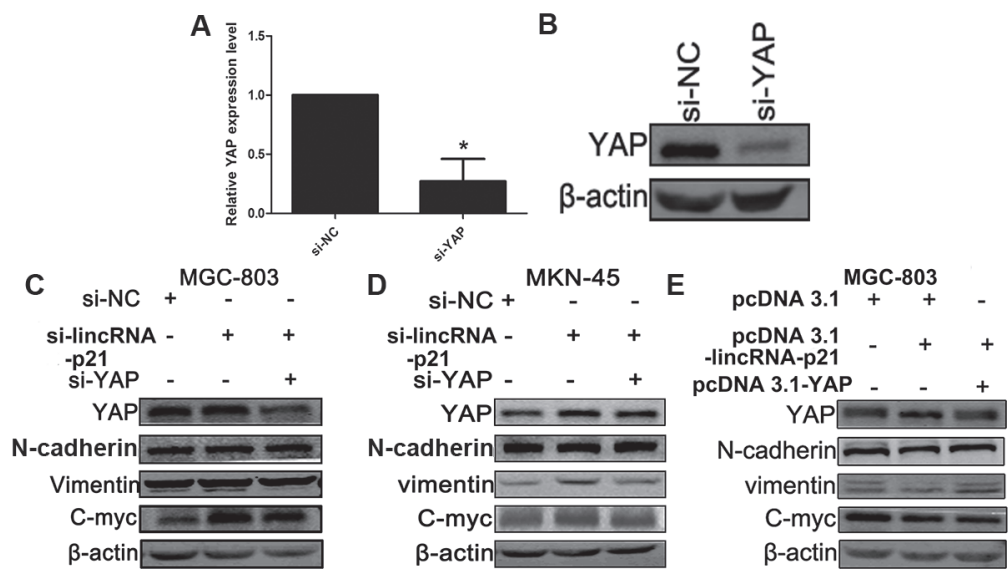

Figure 7: YAP played an important role in the relationship between the lincRNA-p21 and EMT process. The qRT-PCR result (A) and the western-blot result (B) demonstrated the knockdown efficiency of si-YAP. MGC-803 (C) and MKN-45 (D) cells were transfected with si-NC, si-lincRNA-p21 or si-lincRNA-p21/si-YAP. Si-lincRNA-p21/si-YAP group counteracted with the overexpression of YAP induced by the knockdown of lincRNA-p21. The overexpression of N-cadherin, Vimentin and C-myc in si-lincRNA-p21 got reversed. The overexpression experiment showed an opposite result (E). * represents for $\mathrm{p}<0.05$ 
lincRNA-p2 1 was correlated with the higher invasion depth grade, more distant metastasis incidence and more advanced TNM stage, implying lincRNA-p21's role as a potential prognostic biomarker and a therapeutic target for gastric cancer. Meanwhile, we also compared the lincRNA-p21 expression between the GC cell lines and normal gastric mucosal cell line (GES-1). We found decreased lincRNA-p21 expression in GC cell lines, which is consistent with the lower expression levels observed in tumor tissues. To our knowledge, this is the first report of the correlation between lincRNA-p21 expression and gastric cancer progression.

The IncRNAs have been proved to engage in cancer metastasis such as HOTAIR, MALAT1 and HULC [27-29]. Our in vitro experiments showed morphology alteration and up-regulated of $\mathrm{N}$-cadherin and Vimentin expression in si-lincRNA-p21-treated cells. We also found that knocking down lincRNA-p21 expression promoted cell migration and invasion of $\mathrm{GC}$ cell lines. Overexpression of linRNA-p21 verified the correlation between lincRNA-p21 and the EMT process.

YAP, the key component of the Hippo pathway, has been well studied for its implication in organ size control and stem cell differentiation $[19,20]$. The Hippo pathway was originally identified in Drosophila and is comprised of a series of phosphorylation cascades [30-32]. LATS-mediated phosphorylation of YAP downregulates its expression by promoting its cytoplasmic accumulation and the ubiquitination as well as the subsequent proteasomal degradation [33]. However, the expression of YAP is not merely regulated by Hippodependent manner [34-36]. More importantly, the finding of the inter-link between YAP and microRNA biogenesis revealed a potential regulatory role of noncoding RNA in YAP expression [37]. We demonstrated that knockdown of the lincRNA-p21 induced the elevation of both protein and mRNA levels of YAP in a Hippoindependent manner. Furthermore, overexpressing lincRNA-p21 reversed this effect. Intriguingly, we also observed increased YAP expression in both the cytoplasm and nucleus in si-lincRNA-p21 treatments cells. However, the underlying molecular mechanism of the YAP expression alteration induced by changes in lincRNA-p21 expression still needs further elucidation.

In conclusion, the lincRNA-p21 could be regarded as a tumor suppressor in gastric cancer due to its significantly decreased expression in cancer tissues. In addition, the relatively low level of lincRNA-p21 is correlated with higher invasion depth and more distant metastasis incidence rate as well as more advanced TNM stage. Moreover, lincRNA-p21 could impair cancer cell proliferation, migration and invasion ability. This signature is related to the alteration of YAP expression. Most importantly, this elevation is the result of both the up-regulated mRNA level and increased nuclear translocation of YAP.

\section{MATERIALS AND METHODS}

\section{Specimens and relative clinical data}

40 pairs of fresh GC tissues and adjacent nontumor tissues along with the patients' information were acquired from the tissue bank of West China Hospital, Sichuan University, China. The tissues were stored in liquid nitrogen for further RNA extraction. This study was approved by the Research Ethics Committee of West China Hospital. The patients' baseline information was listed in Table 1.

\section{Cell lines}

Five gastric cancer cell lines (MGC-803, MKN-45, BGC-823, MKN-28, SGC-7901) and a normal gastric mucosal cell line (GES-1) were obtained from the cell depository of our laboratory. These cell lines were cultured in Dulbecco's modified Eagle (DMEM) (GIBCO, USA) supplemented with $10 \%$ fetal bovine serum (FBS) (GIBCO, USA) in a humid atmosphere of $5 \% \mathrm{CO} 2$ at $37 \%$.

\section{RNA extraction and quantitative real time-PCR analysis}

The total RNA of patients' tissue specimen and cells were both extracted using the NucleoZOL reagent (MACHEREY-NAGEL, Germany) in accordance to the protocol. The concentration and quality of RNA was detected by NanoDrop. The extracted RNA was converted to cDNA by using PrimeScript ${ }^{\mathrm{TM}}$ RT reagent Kit with gDNA Eraser (Takara, Japan) following the manufacturer's instruction. The cDNA template was mixed with gene specific primers as well as SYBR Green $2 \mathrm{X}$ mixture (Bio-Rad, CA, USA). Quantitative real timepolymerase chain reaction (qRT-PCR) was carried out on Bio-Rad CFX96 Touch (Bio-Rad, USA). The primer sequences were listed in Supplementary Table 1. The expression level of target mRNA was calculated by $2^{-\Delta \Delta \mathrm{CT}}$ values normalized to GAPDH.

\section{RNA interference and plasmid transfection}

The small interfering (si) RNA targeted lincRNA-p21 was designed as same as the known sequence in a former report [38]. Both the si-lincRNA-p21 and a non-specific si-NC as a negative control were both purchased from GenePharma (GenePharma, China). The sequence of the siRNA was listed in Supplementary Table 1. Plasmid used for overexpression, pcDNA 3.1-lincRNA-p21, was constructed and sequencing confirmed by Sangon Company (Sangong, China). MGC803 and MKN-45 were seeded in a 6-well plate $24 \mathrm{~h}$ before transfection at approximately $1 \times 10^{5}$ cells/per well. Then either si-lincRNA-p21 or si-NC was transfected using Lipofectamine 2000 (Invitrogen, USA) under the guidance 
of the protocol. The newly constructed plasmid was extracted by EndoFree Plasmid Mini Kit (CWBIO, China) follow the instruction. We used pcDNA 3.1 as the internal control which was directly obtained from the stock of the lab. 48h later, the transfected cells were harvested for further analysis.

\section{Cell counting Kit-8 assay}

Cell proliferation was tested by Cell Counting Kit-8 (CCK-8) (Dojindo, China) following the manufacturer's instructions. MGC-803 and MKN-45 were seeded in a 96well plate $24 \mathrm{~h}$ before the transfection of si-lincRNA-p21 or si-NC. $100 \mu \mathrm{l}$ of serum free DMEM contains $10 \% \mathrm{CCK}$ 8 reagent was added into each well at $0 \mathrm{~h}$ and $48 \mathrm{~h}$ post transfection respectively and then cultured for $1 \mathrm{~h}$. The absorbance was assessed at a wavelength of 450nm by a microplate reader (Bio-Rad, USA). Experiments were repeated at least three times.

\section{Ethynyl-2-deoxyuridine (EdU) incorporation assay}

Cell proliferation ability was also tested by EdU Apollo DNA in vitro kit (RIBOBIO, China) according to instructions. MGC-803 and MKN-45 were seeded in a 96 -well plate $24 \mathrm{~h}$ before the transfection of either silincRNA-p21 or si-NC. At the $48 \mathrm{~h}$ time point, $100 \mu \mathrm{l}$ of $50 \mu \mathrm{M}$ EdU was added in each well and incubated for 2 $\mathrm{h}$ at $37 \%$. After that, the cells were fixed in $100 \mathrm{ul}$ of $4 \%$ paraformaldehyde (prepared in PBS) for 30 minutes at room temperature. The cells were incubated in $50 \mu \mathrm{l}$ of 2 $\mathrm{mg} / \mathrm{ml}$ glycine for 5 minutes followed by PBS washing. Afterwards, the cells were permeabilized with $1 \%$ TritonX and reacted with 1X Apollo solution for 30 minutes at the room temperature in dark subsequently. Finally, $40 \mu \mathrm{l}$ of Hoechst solution was added in each well and incubated for 15 minutes at room temperature in dark with PBS washing followed. In the end, the cells were visualized and photographed under a fluorescence microscopy. Experiments were repeated at least three times.

\section{Transwell and migration assay}

MGC-803 and MKN-45 were seeded in a 6-well plate $24 \mathrm{~h}$ before the transfection of either si-lincRNA-p21 or si-NC. At the $48 \mathrm{~h}$ time point, the transfected cells were harvested and starved in serum free DMEM and then placed in the upper chambers with or without matrigel coated in duplicate. The lower chambers were filled with DMEM containing 20\% FBS. After wiping off the cells on the upper surface by wet cotton swab, the cells migrated to the lower surface of the chamber were fixed in $4 \%$ paraformaldehyde and stained with $0.5 \%$ crystal violet solution and then photographed under the microscope. Experiments were repeated at least three times.

\section{Wound healing assay}

MGC-803 and MKN-45 were seeded in a 6-well plate $24 \mathrm{~h}$ before the transfection of either si-lincRNA-p21 or si-NC. Right after the transfection, a scratch was made through the center of each well using the 200ul sterile pipette tip. The scratch was observed and pictured at $0 \mathrm{~h}$, $24 \mathrm{~h}, 48 \mathrm{~h}$ post-transfection. Experiments were repeated at least three times.

\section{Western blot assay}

Cellular protein extracts were obtained from cultured cells with RIPA lysis buffer (Bioteke, Beijing, China). The Nuclear and Cytoplasmic Extraction Kit (KeyGEN, Jiangsu, China) was used to prepare cytoplasmic and nuclear extracts separately according to the manufacture's instruction. The protein extracts were separated by $8 \%$ SDSpolyacrylamide gels and transferred electrophoretically onto a PVDF membrane using the Bio-Rad semidry transfer system. Membranes were blocked with 5\% milk overnight and incubated with primary antibodies (diluted in 1:1000) for 2 hours at room temperature. Second antibodies (diluted in 1:100) were added after washing and incubated for 1 hour in dark. After washing again, the membranes were exposed in a dark room using machine. All of the primary antibodies and secondary antibodies were purchased from the Abcam campany.

\section{Fluorescence microscopy}

MGC-803 and MKN-45 cells were transfected by either si-lincRNA-p21 or si-NC 48hrs before plating into 24-well dishes containing $12 \mathrm{~mm}$ glass coverslips. After $24 \mathrm{hrs}$, cells were fixed in 4\% paraformaldehyde and then incubated $1 \mathrm{~h}$ in blocking buffer $(0.5 \%$ triton $\mathrm{X}-100$ and $1 \%$ BSA in PBS) prior to primary antibody staining. Both primary and secondary antibodies were diluted in blocking buffer. Coverslips were incubated with primary antibodies for $1 \mathrm{~h}$ at room temperature followed by $1 \mathrm{~h}$ second antibodies incubation. In the end, diluted DAPI was added on the coverslips. 10 minutes later, coverslips were mounted on the glass slide. Images were captured under Nikon Eclipse 80i (Nikon, Japan) microscope with NIS-Elements software (version 4.30.01).

\section{Statistical analysis}

The analysis between clinicopathological characteristics and lincRNA-p21 expression was performed using SPSS 22.0 software (IBM, USA). The rest data analysis was performed using Graphpad Prism 5.0. Student t test was used for the comparison between two groups and Chisquare test or Fisher's exact test for analyzing the correlation between lincRNA-p21 expression level and patient's clinicopathological characteristics. Quantitative analysis from pictures was performed by ImageJ v1.04 software 
(NIH, Bethesda, MD). All of the $p$ values were two-sided and $p$ values less than 0.05 were considered to be statistically significant.

\section{ACKNOWLEDGMENTS}

We would like to thank the contributions of all authors who participated in this research. This work was supported by the National Natural Science Foundation of China (No. 81572731). The funders had no role in study design, data analysis, preparation of manuscript or decision to publish.

\section{CONFLICTS OF INTEREST}

The authors declare no conflicts interests.

\section{REFERENCES}

1. Ferlay J, Soerjomataram I, Dikshit R, Eser S, Mathers C, Rebelo M, Parkin DM, Forman D, Bray F. Cancer incidence and mortality worldwide: sources, methods and major patterns in GLOBOCAN 2012. Int J Cancer. 2015; 136:E359-386.

2. Herszenyi L, Tulassay Z. Epidemiology of gastrointestinal and liver tumors. Eur Rev Med Pharmacol Sci. 2010; 14:249-258.

3. Kim YI, Kim YW, Choi IJ, Kim CG, Lee JY, Cho SJ, Eom BW, Yoon HM, Ryu KW, Kook MC. Long-term survival after endoscopic resection versus surgery in early gastric cancers. Endoscopy. 2015; 47:293-301.

4. Chen W, Zheng R, Baade PD, Zhang S, Zeng H, Bray F, Jemal A, Yu XQ, He J. Cancer statistics in China, 2015. CA Cancer J Clin. 2016; 66:115-132.

5. Hajjari M, Salavaty A. HOTAIR: an oncogenic long noncoding RNA in different cancers. Cancer Biol Med. 2015; $12: 1-9$

6. Jiao F, Hu H, Han T, Yuan C, Wang L, Jin Z, Guo Z, Wang L. Long noncoding RNA MALAT-1 enhances stem cell-like phenotypes in pancreatic cancer cells. Int J Mol Sci. 2015; 16:6677-6693.

7. Yang F, Bi J, Xue X, Zheng L, Zhi K, Hua J, Fang G. Up-regulated long non-coding RNA H19 contributes to proliferation of gastric cancer cells. FEBS J. 2012; 279:3159-3165.

8. Huarte M, Guttman M, Feldser D, Garber M, Koziol MJ, Kenzelmann-Broz D, Khalil AM, Zuk O, Amit I, Rabani M, Attardi LD, Regev A, Lander ES, et al. A large intergenic noncoding RNA induced by p53 mediates global gene repression in the p53 response. Cell. 2010; 142:409-419.

9. Zhai H, Fesler A, Schee K, Fodstad O, Flatmark K, Ju J. Clinical significance of long intergenic noncoding RNAp21 in colorectal cancer. Clin Colorectal Cancer. 2013; 12:261-266.
10. Yang N, Fu Y, Zhang H, Sima H, Zhu N, Yang G. LincRNA-p21 activates endoplasmic reticulum stress and inhibits hepatocellular carcinoma. Oncotarget. 2015; 6:28151-28163.

11. Zeng $\mathrm{Q}$, Hong $\mathrm{W}$. The emerging role of the hippo pathway in cell contact inhibition, organ size control, and cancer development in mammals. Cancer Cell. 2008; 13:188-192.

12. Zender L, Spector MS, Xue W, Flemming P, CordonCardo C, Silke J, Fan ST, Luk JM, Wigler M, Hannon GJ, $\mathrm{Mu} \mathrm{D}$, Lucito R, Powers S, Lowe SW. Identification and validation of oncogenes in liver cancer using an integrative oncogenomic approach. Cell. 2006; 125:1253-1267.

13. Muramatsu T, Imoto I, Matsui T, Kozaki K, Haruki S, Sudol M, Shimada Y, Tsuda H, Kawano T, Inazawa J. YAP is a candidate oncogene for esophageal squamous cell carcinoma. Carcinogenesis. 2011; 32:389-398.

14. Hall CA, Wang R, Miao J, Oliva E, Shen X, Wheeler T, Hilsenbeck SG, Orsulic S, Goode S. Hippo pathway effector Yap is an ovarian cancer oncogene. Cancer Res. 2010; 70:8517-8525.

15. Wang Y, Dong Q, Zhang Q, Li Z, Wang E, Qiu X. Overexpression of yes-associated protein contributes to progression and poor prognosis of non-small-cell lung cancer. Cancer Sci. 2010; 101:1279-1285.

16. Hong JH, Hwang ES, McManus MT, Amsterdam A, Tian Y, Kalmukova R, Mueller E, Benjamin T, Spiegelman BM, Sharp PA, Hopkins N, Yaffe MB. TAZ, a transcriptional modulator of mesenchymal stem cell differentiation. Science. 2005; 309:1074-1078.

17. Yimlamai D, Christodoulou C, Galli GG, Yanger K, PepeMooney B, Gurung B, Shrestha K, Cahan P, Stanger BZ, Camargo FD. Hippo pathway activity influences liver cell fate. Cell. 2014; 157:1324-1338.

18. Xu MZ, Yao TJ, Lee NP, Ng IO, Chan YT, Zender L, Lowe SW, Poon RT, Luk JM. Yes-associated protein is an independent prognostic marker in hepatocellular carcinoma. Cancer. 2009; 115:4576-4585.

19. Lam-Himlin DM, Daniels JA, Gayyed MF, Dong J, Maitra A, Pan D, Montgomery EA, Anders RA. The hippo pathway in human upper gastrointestinal dysplasia and carcinoma: a novel oncogenic pathway. Int J Gastrointest Cancer. 2006; 37:103-109.

20. Yi BR, Kim TH, Kim YS, Choi KC. Alteration of epithelialmesenchymal transition markers in human normal ovaries and neoplastic ovarian cancers. Int J Oncol. 2015; 46:272-280.

21. Muramaki M, Miyake H, Terakawa T, Kumano M, Sakai I, Fujisawa M. Expression profile of E-cadherin and $\mathrm{N}$-cadherin in non-muscle-invasive bladder cancer as a novel predictor of intravesical recurrence following transurethral resection. Urol Oncol. 2012; 30:161-166.

22. Wang G, Li Z, Zhao Q, Zhu Y, Zhao C, Li X, Ma Z, Li $X$, Zhang Y. LincRNA-p21 enhances the sensitivity of radiotherapy for human colorectal cancer by targeting the 
Wnt/beta-catenin signaling pathway. Oncol Rep. 2014; 31:1839-1845.

23. Spurlock CF 3rd, Tossberg JT, Matlock BK, Olsen NJ, Aune TM. Methotrexate inhibits NF-kappaB activity via long intergenic (noncoding) RNA-p21 induction. Arthritis Rheumatol. 2014; 66:2947-2957.

24. Mo JS, Park HW, Guan KL. The Hippo signaling pathway in stem cell biology and cancer. EMBO reports. 2014; 15:642-656.

25. Eades G, Zhang YS, Li QL, Xia JX, Yao Y, Zhou Q. Long non-coding RNAs in stem cells and cancer. World J Clin Oncol. 2014; 5:134-141.

26. Wang KC, Chang HY. Molecular mechanisms of long noncoding RNAs. Mol Cell. 2011; 43:904-914.

27. Ji P, Diederichs S, Wang W, Boing S, Metzger R, Schneider PM, Tidow N, Brandt B, Buerger H, Bulk E, Thomas M, Berdel WE, Serve H, Muller-Tidow C. MALAT-1, a novel noncoding RNA, and thymosin beta4 predict metastasis and survival in early-stage non-small cell lung cancer. Oncogene. 2003; 22:8031-8041.

28. Gupta RA, Shah N, Wang KC, Kim J, Horlings HM, Wong DJ, Tsai MC, Hung T, Argani P, Rinn JL, Wang Y, Brzoska P, Kong B, et al. Long non-coding RNA HOTAIR reprograms chromatin state to promote cancer metastasis. Nature. 2010; 464:1071-1076.

29. Panzitt K, Tschernatsch MM, Guelly C, Moustafa T, Stradner M, Strohmaier HM, Buck CR, Denk H, Schroeder R, Trauner M, Zatloukal K. Characterization of HULC, a novel gene with striking up-regulation in hepatocellular carcinoma, as noncoding RNA. Gastroenterology. 2007; 132:330-342.

30. Zhao B, Li L, Wang L, Wang CY, Yu J, Guan KL. Cell detachment activates the Hippo pathway via cytoskeleton reorganization to induce anoikis. Genes Dev. 2012; 26:54-68.
31. Zhao B, Wei X, Li W, Udan RS, Yang Q, Kim J, Xie J, Ikenoue T, Yu J, Li L, Zheng P, Ye K, Chinnaiyan A, et al. Inactivation of YAP oncoprotein by the Hippo pathway is involved in cell contact inhibition and tissue growth control. Genes Dev. 2007; 21:2747-2761.

32. Hao Y, Chun A, Cheung K, Rashidi B, Yang X. Tumor suppressor LATS1 is a negative regulator of oncogene YAP. J Biol Chem. 2008; 283:5496-5509.

33. Zhao B, Li L, Tumaneng K, Wang CY, Guan KL. A coordinated phosphorylation by Lats and $\mathrm{CK} 1$ regulates YAP stability through SCF(beta-TRCP). Genes Dev. 2010; 24:72-85.

34. Yu FX, Zhao B, Panupinthu N, Jewell JL, Lian I, Wang LH, Zhao J, Yuan H, Tumaneng K, Li H, Fu XD, Mills GB, Guan KL. Regulation of the Hippo-YAP pathway by G-protein-coupled receptor signaling. Cell. 2012; 150:780-791.

35. Azzolin L, Panciera T, Soligo S, Enzo E, Bicciato S, Dupont S, Bresolin S, Frasson C, Basso G, Guzzardo V, Fassina A, Cordenonsi M, Piccolo S. YAP/TAZ incorporation in the beta-catenin destruction complex orchestrates the Wnt response. Cell. 2014; 158:157-170.

36. Fujii M, Toyoda $T$, Nakanishi $H$, Yatabe $Y$, Sato A, Matsudaira $\mathrm{Y}$, Ito $\mathrm{H}$, Murakami $\mathrm{H}$, Kondo $\mathrm{Y}$, Kondo E, Hida T, Tsujimura T, Osada H, Sekido Y. TGF-beta synergizes with defects in the Hippo pathway to stimulate human malignant mesothelioma growth. J Exp Med. 2012; 209:479-494.

37. Mori M, Triboulet R, Mohseni M, Schlegelmilch K, Shrestha K, Camargo FD, Gregory RI. Hippo signaling regulates microprocessor and links cell-density-dependent miRNA biogenesis to cancer. Cell. 2014; 156:893-906.

38. Yang F, Zhang H, Mei Y, Wu M. Reciprocal regulation of HIF-1alpha and lincRNA-p21 modulates the Warburg effect. Mol Cell. 2014; 53:88-100. 